\title{
Multiple cholinergic differentiation factors are present in footpad extracts: comparison with known cholinergic factors
}

\author{
M. S. RAO ${ }^{1,2}$, PAUL H. PATTERSON ${ }^{1}$ and S. C. LANDIS ${ }^{2, *}$ \\ ${ }^{1}$ Division of Biology, California Institute of Technology, Pasadena, CA 91125, USA \\ ${ }^{2}$ Department of Neurosciences, Case Western University, Cleveland, OH 44106, USA \\ *Author for correspondence
}

\section{Summary}

Sweat glands in rat footpads contain a neuronal differentiation activity that switches the phenotype of sympathetic neurons from noradrenergic to cholinergic during normal development in vivo. Extracts of developing and adult sweat glands induce changes in neurotransmitter properties in cultured sympathetic neurons that mimic those observed in vivo. We have characterized further the factors present in the extract and compared their properties to those of known cholinergic factors. When assayed on cultured rat sympathetic neurons, the major activities in footpad extracts from postnatal day 21 rat pups that induce choline acetyltransferase (ChAT) and vasoactive intestinal peptide (VIP) and reduce catecholamines and neuropeptide $Y$ (NPY) are associated with a soluble protein of $22-26 \times 10^{3}$ $M_{\mathrm{r}}$ and a pI of 5.0. These properties are similar to those of ciliary neurotrophic factor (CNTF). Moreover, the purified fraction from footpads has ciliary neurotrophic activity. Antibodies to CNTF that immunoprecipitate all differentiation activity from sciatic nerve extracts, a rich source of CNTF, immunoprecipitate $80 \%$ of the cholinergic activity in the footpad extracts, $50 \%$ of the VIP and $20 \%$ of the NPY activities. Neither CNTF protein nor CNTF mRNA, however, can be detected in immunoblot and northern analysis of footpads even though both CNTF protein and mRNA are evident in sciatic nerve. CNTF-immunoreactivity is associated with a sparse plexus of sensory fibers in the footpad but not with sweat glands or the Schwann cells associated with them. In addition, in situ hybridization studies with oligonucleotide probes failed to reveal CNTF mRNA in sweat glands. Comparison of the sweat gland differentiation activity with the cholinergic differentiation factor from heart cells (CDF; also known as leukemia inhibitory factor or LIF) suggests that most of the cholinergic activity in foot pads is biochemically distinct from CDF/LIF. Further, antibodies that block the activity of CDF/LIF purified from heart-cell-conditioned medium do not block the ChAT-inducing activity present in footpad extracts of postnatal day 8 animals. A differentiation factor isolated from skeletal muscle did not induce cholinergic properties in sympathetic neuron cultures and therefore is unlikely to be the cholinergic differentiation factor produced by sweat glands. Taken together, our data suggest that there are at least two differentiation molecules present in the extracts and that the major cholinergic activity obtained from footpads is related to, but distinct from, CNTF. The second factor remains to be characterized. In addition, CNTF associated with sensory fibers may make a minor contribution to the cholinergic inducing activity present in the extract.

Key words: CDF/LIF, CNTF, sympathetic neurons, neurotransmitter plasticity, neural crest.

\section{Introduction}

While the sympathetic innervation of adult rat sweat glands is cholinergic, the axons that initially innervate the sweat gland are noradrenergic (Landis and Keefe, 1983; Leblanc and Landis, 1986; Stevens and Landis, 1987). Thus, there is a switch in the neurotransmitter phenotype of these sympathetic neurons. Cross-innervation studies in vivo indicate that this switch is target-directed. Neurons that normally innervate sweat glands fail to acquire cholinergic properties when they are made to innervate the parotid gland, a target whose sympathetic innervation is ordinarily nora- drenergic (Schotzinger and Landis, 1990). Furthermore, normally noradrenergic sympathetic neurons that innervate hairy skin become cholinergic when they are made to innervate sweat glands (Schotzinger and Landis, 1988). Acquisition of immunoreactivity for vasoactive intestinal peptide (VIP), a neuropeptide present in mature sweat gland innervation, is also target-dependent (Landis et al., 1988; Schotzinger and Landis, 1988; Stevens and Landis, 1990 and unpublished observations). Although the evidence for target control of transmitter phenotype is compelling, the identity of the molecules involved is not known.

We and others previously found that extracts of rat foot- 
pads contain factor(s) that induce choline acetyltransferase (ChAT) and VIP, and reduce catecholamine and neuropeptide Y (NPY) content in cultured sympathetic neurons (Rao and Landis, 1990; Rohrer, 1992). This activity was not detected in extracts of parotid gland or hairy skin, tissues that receive noradrenergic sympathetic innervation. The cholinergic differentiation activity is evident in developing footpads as early as postnatal day 5 (P5) and increases over a period of two weeks to reach adult levels. Therefore, it is present at the appropriate time in development to be responsible for the target-mediated noradrenergic-to-cholinergic switch.

Several purified proteins elicit the same noradrenergicto-cholinergic switch in cultured sympathetic neurons and are therefore candidates for the differentiation signal in sweat glands. The cholinergic differentiation factor (CDF), originally purified from heart-cell-conditioned medium (Patterson and Chun, 1977; Fukada, 1985), is identical to leukemia inhibitory factor (LIF; Yamamori et al., 1989). Recombinant CDF/LIF also influences neuropeptide expression in cultured sympathetic neurons in a manner very similar to that of the footpad factor (Rao and Landis, 1990; Nawa et al., 1990). Studies using the reverse transcriptase/polymerase chain reaction (RT-PCR) revealed that CDF/LIF mRNA is selectively localized in postnatal rat footpads (Yamamori, 1991). Another candidate for the sweat gland cholinergic factor is ciliary neurotrophic factor (CNTF; Lin et al., 1989; Stockli et al., 1989). This protein regulates cholinergic, noradrenergic and neuropeptide properties in cultured sympathetic neurons in the same fashion as CDF/LIF (Ernsberger et al., 1989; Saadat et al., 1989; Rao et al., 1992a). A third cholinergic factor, MANS (membrane associated neurotransmitter stimulating factor), was partially purified from rat spinal cord (Wong and Kessler, 1987; Adler et al., 1989). MANS appears to be related to CNTF since it supports the survival of chick ciliary neurons (Rao et al., 1990) and antibodies to CNTF immunoprecipitate the cholinergic inducing activity of MANS and detect a band of $24 \times 10^{3}$, the relative molecular mass of CNTF (Carnow et al., 1985; Manthorpe et al., 1986), on immunoblots of MANS preparations (Rao et al., 1992b). Finally, a cholinergic differentiation factor, which is biochemically distinct from CDF/LIF and CNTF, has been purified from skeletal muscle. It enhances the survival of motor neurons in vitro and in vivo and increases levels of ChAT in spinal cord cultures (McManaman et al., 1988, 1989, 1990). The ability of the skeletal muscle cholinergic differentiation factor to induce cholinergic properties in sympathetic neurons has not been tested. Since CDF/LIF and CNTF, two of the factors that induce cholinergic function in sympathetic neurons, affect motor neurons (Geiss and Weber, 1984; Martinou et al., 1989; 1992; Sendtner et al., 1990; Arakawa et al., 1990), it seemed plausible that the skeletal muscle cholinergic factor would also induce cholinergic function in sympathetic neurons.

To determine if any of the known cholinergic factors is present in sweat gland, we further characterized the cholinergic inducing activity from footpad extracts and compared its properties with $\mathrm{CDF} / \mathrm{LIF}, \mathrm{CNTF}$ and the cholinergic factor from skeletal muscle. We found that most of the differentiation activity present in footpads of young rats is associated with a molecule(s) that is immunoprecipitated by antibodies that recognize CNTF. We did not, however, detect either CNTF mRNA or protein in footpads in immunoblot, in northern blot or in situ hybridization analyses, suggesting that the major differentiation factor present in the extracts and recognized by CNTF antibodies is similar, but not identical, to CNTF. The second differentiation activity is as yet uncharacterized.

\section{Materials and methods}

Cell culture reagents were obtained from Gibco (Grand Island, NY). 24- and 96-well culture plates were purchased from Corning (Corning, NY). Affi-gel 10 was obtained from Bio-rad (Richmond, CA.). The Centricon filters were purchased from Amicon (Danvers, MA). $\left[{ }^{3} \mathrm{H}\right]$ acetyl-CoA was purchased from New England Nuclear (Wilmington, DE). T4 polynucleotide kinase and 3' end terminal transferase were obtained from (BRL), gamma ${ }^{32} \mathrm{P}$ from Amersham (Arlington Heights, IL) and ${ }^{35} \mathrm{~S}-\mathrm{dATP}$ from NEN. The VIP RIA kit was obtained from INCSTAR (Minneapolis, MN) and the NPY RIA kit and rat NPY were obtained from Peninsula (Belmont, CA). Dispase was purchased from Boehringer-Mannheim (Indianapolis, IN) and collagenase from Worthington Biochemicals (Freehold, NJ). Genescreen membranes were obtained from NEN. Vectastain ABC immunoperoxidase kits were obtained from Vector Laboratories (Burlingame, CA) and diaminobenzidine from Polysciences (Warrington, PA). The rabbit antiserum against CGRP and the avidin-Texas red conjugates were obtained from Amersham. Other chemicals were purchased from Sigma Chemical Co. (St Louis, MO). Nerve Growth Factor (NGF) was provided by Dr Ken Neet (Department of Biochemistry, Chicago Medical School). An antiserum that blocks the activity of CDF/LIF was obtained from R \& D Systems (St Paul, $\mathrm{MN}$ ) and an antiserum generated against recombinant rat CNTF was the kind gift of Dr Morrissey at Regeneron Pharmaceuticals (Tarrytown, NY). Animals were obtained from Zivic Miller (Zelienople, PA).

\section{Cholinergic factors}

Recombinant rat CNTF, produced in $E$. coli, was obtained from Regeneron Pharmaceuticals (Tarrytown, NY). Recombinant human LIF was obtained from AMGEN (Thousand Oaks, CA). CDF/LIF purified from heart-cell-conditioned medium was provided by Dr Keiko Fukada (SUNY Downstate, New York, NY; Fukada et al., 1991). The cholinergic differentiation factor from skeletal muscle was obtained from Dr J. McManaman (Baylor College of Medicine, Houston, TX); the HAP7 fraction was used for all experiments (McManaman et al., 1989). Footpad extracts were prepared as described previously with the exception that the whole footpad was used rather than the gland-rich core (Rao and Landis, 1990). Unless otherwise stated, the DEAE fraction of extracts from 21-day-old rats was used. Sciatic nerve extracts were prepared from adult rats as described previously (Rao et al., 1990). The $0.25 \mathrm{M} \mathrm{NaCl}$ eluate of the DEAE column was used for all the experiments.

\section{Column chromatography}

The soluble fractions of homogenates of footpads of 21-day-old rats were concentrated (tenfold) with a centriprep microconcentrator (Amicon), and the final sample buffer contained $10 \mathrm{mM}$ phosphate, $\mathrm{pH} 7.0 .10 \mathrm{mg}$ protein was chromatographed on a MonoQ column (Pharmacia) using a Whatman HPLC. Fractions of $0.5 \mathrm{ml}$ were collected while eluting with a linear gradient to $0.1 \mathrm{M} \mathrm{NaCl}$ (Fig. 1), and assayed for cholinergic activity on cul- 

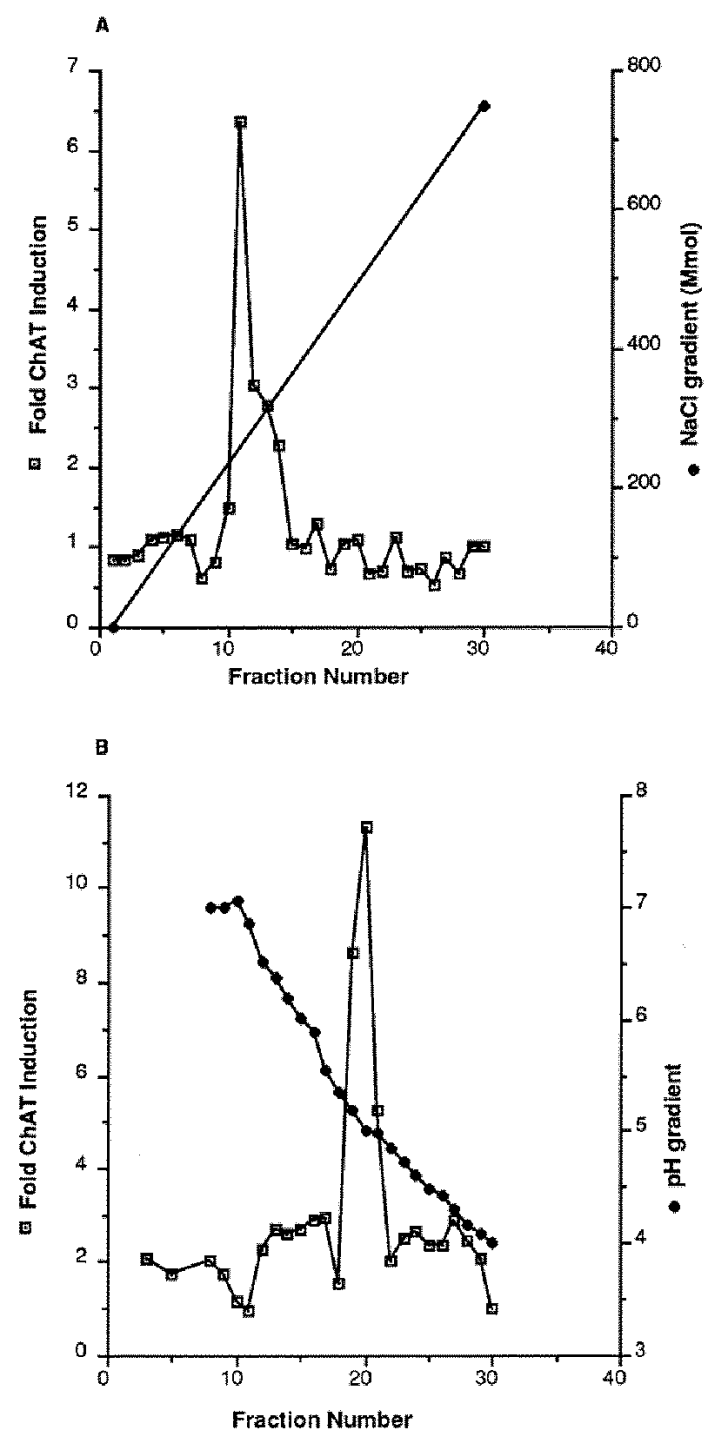

Fig. 1. (A) The major cholinergic inducing activity in footpad extracts is an acidic protein. Soluble protein extracted from footpads of 21-day-old rats was chromatographed on a Mono Q anion exchange column and $0.5 \mathrm{ml}$ fractions were collected, concentrated and added to sympathetic neuron cultures. 7 days after the addition of the extracts, neurons were homogenized and aliquots assayed for ChAT activity. The points represent the averages of duplicate determinations. The data are expressed as the fold induction of ChAT activity compared to that present in cultures grown without added extract. (B) The major cholinergic inducing activity in footpad extracts has a pI of 5.0. The peak fraction (12) from the Mono Q column was chromatographed on a Mono $\mathrm{P}$ chromatofocussing column. Fractions were concentrated and added to sympathetic neuron cultures. 7 days later, the neurons were homogenized and aliquots were assayed for ChAT activity. Points are averages of duplicate determinations. The data are expressed as the fold induction of ChAT activity compared to that present in cultures grown without added extract.

tured sympathetic neurons. Active fractions from several runs of the MonoQ column were pooled, concentrated and chromatographed on a MonoP HR 5/5 column (Pharmacia) that had been equilibrated with $25 \mathrm{mM}$ bis-Tris, $\mathrm{pH}$ 7.1. Elution was with a polybuffer at $\mathrm{pH} 4.0$ containing saturating levels of iminodiacetic acid. The flow-through and eluted fractions were adjusted to neutral $\mathrm{pH}$ and stored at $-70^{\circ} \mathrm{C}$. Control experiments showed that the polybuffer did not affect the growth or differentiation of neuronal cultures. Active fractions ( $\mathrm{pH}$ 4.9-5.2) were pooled, concentrated and chromatographed on a Sepharose 12 column (Pharmacia) equilibrated in $0.1 \mathrm{M} \mathrm{NaCl}, 10 \mathrm{mM}$ phosphate, $\mathrm{pH} 7.0$. Fractions of $0.5 \mathrm{ml}$ were collected and assayed for biological activity. Protein standards were chromatographed under identical conditions to determine the approximate sizes of proteins collected in the various fractions.

\section{Elution of proteins from SDS-PAGE}

The active fractions from the MonoP column were pooled, concentrated ten fold and subjected to preparative polyacrylamide gel electrophoresis in sodium dodecyl sulfate (SDS-PAGE). A $15 \%$ acrylamide gel was used in a Biorad apparatus. Regions of the gel of appropriate relative molecular mass were cut out and loaded on a Biorad electroeluter. Elution was at $10 \mathrm{~mA} /$ tube for 4 hours in a buffer containing $50 \mathrm{mM}$ ammonium bicarbonate and $0.1 \%$ SDS. Samples were concentrated and SDS precipitated by adding $\mathrm{KCl}$ to a final concentration of $0.1 \mathrm{M}$, as described by Suzuki and Terada (1988). Bovine serum albumin (1\%; BSA) was added to the samples, which were then dialyzed on a Centricon apparatus $\left(10 \times 10^{3} M_{\mathrm{r}}\right.$ size cutoff) to remove excess $\mathrm{KCl}$. Samples were stored at $-70^{\circ} \mathrm{C}$ until used.

\section{Cell culture}

Cultures of rat sympathetic neurons were prepared as described by Hawrot and Patterson (1979). Neurons were dissociated enzymatically with Dispase $(5 \mathrm{mg} / \mathrm{ml})$ and collagenase $(1 \mathrm{mg} / \mathrm{ml})$ and plated in 96-well polylysine-laminin-coated plates. 1000-2000 neurons were plated per well. Neurons were grown in $\mathrm{L} 15-\mathrm{CO}_{2}$ medium with $7 \mathrm{~S}$ NGF (100 ng/ml), $10 \mu \mathrm{M}$ cytosine arabinoside and $5 \%$ adult rat serum, and the medium changed every second day. The cholinergic factors and column fractions were diluted in growth medium, sterilized by passage through a $0.2 \mu \mathrm{m}$ filter and added to the neurons on the third day of culture. Neurons were harvested for assay between days 9 and 14 of culture.

Ciliary ganglia were dissected from embryonic day 8 (E8) chicks, dissociated and plated in DMEM with $10 \%$ fetal calf serum as described by Varon et al. (1979). 1000-2000 neurons were plated directly into medium in 96-well culture plates coated sequentially with polylysine and laminin. The cultures were incubated for 24 hours at $37^{\circ} \mathrm{C}$, and fixed with $2 \%$ glutaraldehyde as described by Barbin et al. (1984). The number of surviving neurons was determined by counting phase bright cells with neurites.

\section{Assays of neurotransmitter properties}

The induction of cholinergic function was determined by assaying ChAT activity in homogenates essentially according to the method of Fonnum (1969). To increase the sensitivity of the assay, an incubation period of an hour was used. All activity was inhibitable by $500 \mu \mathrm{M}$ naphthylvinyl pyridine, a specific inhibitor of ChAT activity (Smith et al., 1967; White and Wu, 1973).

Tyrosine hydroxylase (TH) content was assayed by immunoblot. Aliquots of cultured neuron extracts ( $10^{4}$ neurons) were subjected to SDS-PAGE on a $15 \%$ minigel and the proteins transferred to nitrocellulose overnight at $30 \mathrm{~mA}$. The blots were blocked (5\% defatted milk in Tris-buffered saline, $\mathrm{pH}$ 7.2) and then incubated for two hours with a monoclonal antibody that recognizes tyrosine hydroxylase (TH; 1:1000 dilution, the kind gift of Dr Ann Acheson, Edmonton, Alberta, Canada; Rohrer et al., 1986). The blots were stained and developed as described for the CNTF immunoblots.

Neuropeptide levels were determined by radioimmunoassay. Neuronal cultures $\left(10^{4}\right.$ neurons) were rinsed once with PBS and homogenized in $100 \mu \mathrm{l} 2 \mathrm{M}$ acetic acid. After boiling for five 
minutes, samples were centrifuged for 1 minute in an Eppendorf microfuge. Supernatants were dried under vacuum and stored at $-70^{\circ} \mathrm{C}$ for subsequent assay. VIP was assayed using primary antibodies previously demonstrated to show minimal crossreactivity with other peptides. NPY was assayed by the delayed tracer method as specified in the kit using antibodies, standards and labeled tracer from Amersham. The standard curve was produced with rat NPY.

\section{Immunoprecipitation}

For the immunoprecipitation experiments in which the biological activity of the factors was tested, aliquots of CDF/LIF and extracts of sciatic nerve from adult rats or footpads from 21-day-old animals sufficient for a cell culture assay were added to buffer (phosphate-buffered saline [PBS], pH 7.3 with $2 \%$ BSA, $0.2 \%$ Triton $\mathrm{X}-100$, and $0.02 \%$ polyethylene glycol 6000 ). A polyclonal antiserum generated against recombinant rat CNTF (obtained from Dr Donna Morissey, Regeneron Pharmaceuticals) was added to each vial to a final concentration of $10 \mu \mathrm{M}$. After an overnight incubation, the antigen-antibody complex was absorbed to $10 \mu \mathrm{l}$ of protein A Sepharose (in the same buffer) for a further two hours at room temperature. The bound complexes were separated by centrifugation and the supernatant was diluted into $\mathrm{L} 15-\mathrm{CO}_{2}$ medium and used for cell culture assays. To ensure that the loss of activity was due to a specific effect of the antibody, aliquots of the cholinergic factors were incubated without the antibody and treated as described above.

\section{Antibody blocking of CDF/LIF activity}

A polyclonal antiserum generated in rabbit against CDF/LIF purified from heart-cell-conditioned medium (the kind gift of Dr Keiko Fukada, SUNY Downstate, New York, NY; Fukada and Towle, 1992) was used for antibody blocking experiments. Sympathetic neurons were grown in Optimem defined medium with $100 \mathrm{ng} / \mathrm{ml}$ NGF. On the second day after plating, extract from postnatal day 8 rat pups or CDF/LIF purified from heart cell conditioned medium $(20 \mathrm{ng} / \mathrm{ml})$ were added to the cultures. Antiserum was added to a final dilution of 1:15 which had been previously determined to block ChAT induction in response to treatment with purified CDF/LIF. Cultures were fed every fourth day and harvested on the ninth day after initiating antibody treatment. Samples were assayed for ChAT activity as described above. Two controls were performed to examine the specificity of the antiserum. Cultures were grown in the same dilution of antiserum but in the absence of $\mathrm{CDF} / \mathrm{LIF}$ or in the presence of antiserum and sciatic nerve extract $(25 \mu \mathrm{g} / \mathrm{ml})$.

\section{CNTF immunoblots}

Aliquots of extracts were run on a $15 \%$ SDS-PAGE minigel (Biorad) and the proteins were blotted onto nitrocellulose (overnight, $30 \mathrm{~mA})$. The blots were blocked $(5 \%$ defatted milk in Tris-buffered saline, $\mathrm{pH}$ 7.2) and then incubated for two hours with anti-CNTF antiserum (1:1000 dilution) or with the antibody preincubated with $10 \mu \mathrm{M}$ CNTF. The blots were then sequentially incubated with a biotinylated secondary antibody (1 hour at room temperature) and avidin conjugated to alkaline phosphatase $(0.5$ hour). The bound enzyme was detected with NBT (nitroblue tetrazolium) and BCIP (bromo-chloro-indoyl phosphate) in $10 \mathrm{mM}$ bicarbonate buffer, $\mathrm{pH}$ 9.5. After color development, the reaction was stopped by rinsing in distilled water.

\section{Northern blots}

Total RNA was prepared from liver, foot pad and sciatic nerve using the single step guanidinium-isothiocyanate method (Chom- czynski and Sacchi, 1987). The RNA was denatured on a $1.5 \%$ agarose gel containing $12.2 \mathrm{M}$ formaldehyde and blotted onto Genescreen Plus membranes. Blots were probed with a 45 base pair antisense oligonucleotide probe for rat CNTF (bases 99-144). The probe was labeled at the $5^{\prime}$ end using $\mathrm{T} 4$ polynucleotide kinase and gamma ${ }^{32} \mathrm{P}$. Hybridization was performed at $42^{\circ} \mathrm{C}$ for 48 hours in buffer consisting of $1 \mathrm{M} \mathrm{NaCl}, 30 \%$ deionized formamide, and $10 \%$ dextran sulfate. Blots were sequentially washed at $50^{\circ} \mathrm{C}$ in $0.1 \times \mathrm{SSC}$ and exposed to Kodak XAR-5 film for 3-5 days.

\section{In situ hybridization}

The probe used in the northern blot experiments was also used for in situ hybridization after labeling at the $3^{\prime}$ end using terminal transferase and ${ }^{35} \mathrm{~S}$-dATP. The sense probe to the same region was used as a control. The in situ hybridization method was as described by Siegel (1989). Briefly, fresh-frozen tissue sections were fixed in $4 \%$ formaldehyde for 5 minutes, rinsed three times in PBS, incubated in acetic anhydride/triethanolamine for $10 \mathrm{~min}-$ utes, rinsed two times briefly in $2 \times \mathrm{SSC}(0.3 \mathrm{M} \mathrm{NaCl}, 0.03 \mathrm{M}$ sodium citrate, $\mathrm{pH} 7.3$ ) and finally dehydrated through a series of graded alcohols. The slides were air-dried and processed for hybridization. After a one hour prehybridization incubation (4×SSC, $50 \%$ formamide, $500 \mu \mathrm{g} / \mathrm{ml}$ sheared single-stranded DNA, $250 \mu \mathrm{g} / \mathrm{ml}$ yeast tRNA, $1 \times$ Denharts solution [0.02\% Ficoll, $0.02 \%$ polyvinylpyrrolidone, $0.02 \%$ bovine serum albumin], and $10 \%$ dextran sulfate), the blots were rinsed with $2 \times \mathrm{SSC}$ and radioactive probe, diluted in hybridization buffer, was applied at a concentration of $10^{6} \mathrm{cts} /$ minute/slide. Parafilm coverslips were placed over the sections and hybridization was carried out in humid chambers overnight at room temperature. After washing, the sections were dipped in Kodak NTB-3 emulsion and exposed for 6 weeks. Sections were developed, fixed and counterstained with ethidium bromide or cresyl violet.

\section{Immunocytochemistry}

The distribution of CNTF immunoreactivity was examined in footpads and in hairy skin. Rats were killed with ether and perfused through the heart with $4 \%$ paraformaldehyde in $0.1 \mathrm{M}$ phosphate

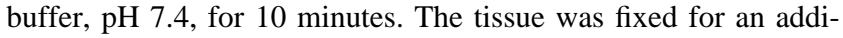
tional hour by immersion in the paraformaldehyde solution. After rinsing, the tissue was equilibrated with $30 \%$ sucrose in PBS. 15 $\mu \mathrm{m}$ cryostat sections were collected and thaw mounted onto gelatin-coated slides. The sections were incubated overnight at room temperature with a primary antiserum generated in rabbit against a synthetic peptide from CNTF (amino acids 181-200; Rende et al., 1992; the kind gift of Dr Manthorpe) diluted 1:2000 in dilution buffer $(0.5 \mathrm{M} \mathrm{NaCl}, 0.01 \mathrm{M}$ phosphate buffer, $3 \%$ bovine serum albumin, $0.1 \%$ sodium azide and $0.3 \%$ Triton $\mathrm{X}$ 100). The sections were rinsed, incubated in biotinylated secondary antibody for 2 hours, rinsed and then incubated with the Vectastain avidin-horseradish peroxidase (HRP) complex for 2 hours. After rinsing, the bound antibody was visualized with $3,3^{\prime}$ diaminobenezidine $(0.5 \mathrm{mg} / \mathrm{ml})$ in $0.1 \mathrm{M}$ Tris buffer, $\mathrm{pH} 7.2$, containing $0.02 \% \mathrm{H}_{2} \mathrm{O}_{2}$. Optimal reaction product developed within 15 seconds to 2 minutes. To control for antiserum specificity, sections were incubated without primary antibody or with primary antiserum preabsorbed with $20 \mu \mathrm{g} / \mathrm{ml}$ of recombinant CNTF. In a second set of experiments, double labeling for CNTF and calcitonin gene-related peptide (CGRP), a neuropeptide present in sensory fibers in footpad epidermis (Kruger et al., 1989), was performed. Sections were incubated with the CNTF antiserum, rinsed, incubated with biotinylated secondary antiserum and then with avidin conjugated to Texas red. A second series of incubations was then carried out to detect CGRP. The sections were incubated with a CGRP antiserum raised in rabbit, rinsed, incubated in goat anti-rabbit conjugated to fluorescein, rinsed and mounted in 
PBS/glycerin. The sequential use of two rabbit antisera could yield cross-reactivity between the secondary antisera. Control sections were incubated with the goat anti-rabbit fluorescein after the avidin-conjugated Texas red without the second primary antiserum and no specific fluorescein staining was evident.

\section{Results}

\section{Biochemical characterization of footpad factor}

Footpads from postnatal day 21 rats were homogenized and the extract was applied to a Mono $Q$ anionic exchange column. This age was selected because previous studies had shown that footpads from 21-day-old rats had the greatest amount of cholinergic inducing activity on a per protein basis (Rao and Landis, 1990). As shown in Fig. 1A, the cholinergic activity bound to the column and was eluted as a single peak at $0.25 \mathrm{mM} \mathrm{NaCl}$. This chromatographic profile is similar to that of CNTF and the cholinergic factor from skeletal muscle (Manthorpe et al., 1986; McManaman et al., 1988, 1989), but is different from that of the basic protein, CDF/LIF (Fukada, 1985). Similar results were obtained with extracts from rats 28 days and older (data not shown). The Mono Q peak fraction was then chromatographed on a Mono P column. The cholinergic inducing activity eluted between $\mathrm{pH} 4.8$ and 5.2 (Fig. 1B). The $\mathrm{pH}$ of the peak fraction, 5.0, is similar to the value reported for CNTF purified from rat sciatic nerve (Manthorpe et al., 1986). In each case, cholinergic inducing activity was detected in a single peak.

To determine the approximate relative molecular masses of the cholinergic inducing activity, peak fractions from the Mono P column were chromatographed on a Sepharose 12 column. The activity eluted in a peak between the $16 \times 10^{3}$ $M_{\mathrm{r}}$ and $32 \times 10^{3} M_{\mathrm{r}}$ size markers (Fig. 2). This relative molecular mass is comparable to those of MANS (Wong and Kessler, 1987), CNTF (Manthorpe et al., 1986) and deglycosylated CDF/LIF (Fukada, 1985). The relative molecular mass of the footpad cholinergic activity was determined more accurately by SDS-PAGE. Purified material from the Mono P column was subjected to SDS-PAGE. Slices of the gel corresponding to $22-26 \times 10^{3}$ and $26-32 \times 10^{3} M_{\mathrm{r}}$ were removed and the proteins eluted with an electroeluter. As shown in Fig. 3A, most of the cholinergic activity was recovered in the $22-26 \times 10^{3} M_{\mathrm{r}}$ fraction.

The SDS-PAGE fractions were also tested for their effects on VIP, TH and NPY expression. As in the case of the cholinergic inducing activity, most of the TH- and NPYsuppressing and VIP-inducing activities were in the 22$26 \times 10^{3} M_{\mathrm{r}}$ fraction (Fig. 3B-D).

Comparison of the foot pad cholinergic activity with CNTF Since the size and $\mathrm{pI}$ of the cholinergic differentiation activity in the footpad extract were very similar to those of CNTF, we examined the ability of SDS-PAGE-purified material to support the survival of E8 chick ciliary neurons in culture, the original assay for CNTF activity (Barbin et al., 1984; Manthorpe et al., 1986). Fig. 4 shows that the $22-26 \times 10^{3} M_{\mathrm{r}}$ fraction purified from footpad extract contained a significant level of ciliary neurotrophic activity. Thus, this fraction contained not only cholinergic and pep-

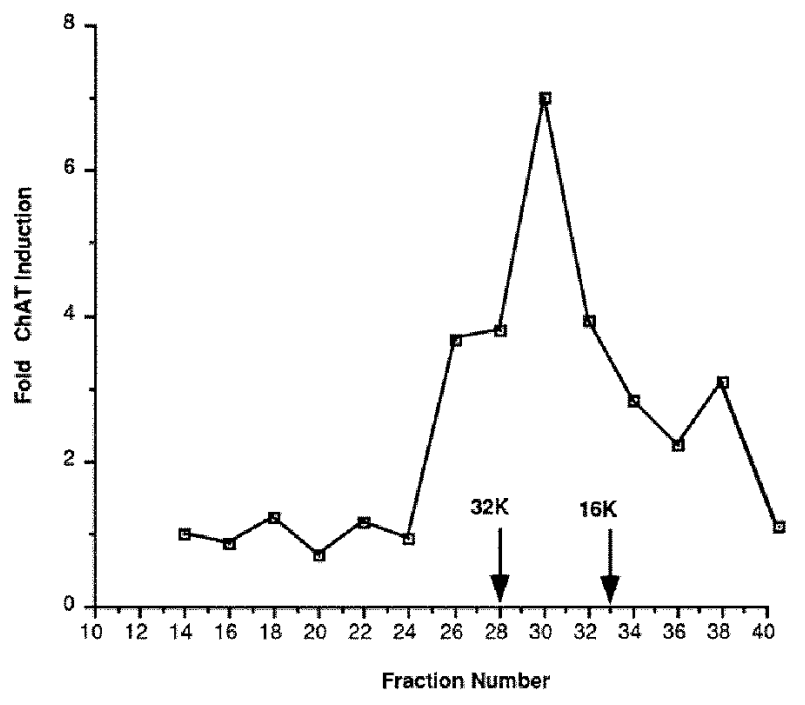

Fig. 2. The major cholinergic activity in footpad extracts is a low relative molecular mass protein. The peak fraction from the Mono $\mathrm{P}$ column was chromatographed on a Sepharose 12 column.

Fractions were concentrated and added to sympathetic neuron cultures. 7 days after the addition of the extracts, the neurons were homogenized and aliquots were assayed for ChAT activity. The points represent the averages of duplicate determinations. The data are expressed as the fold induction of ChAT activity compared to that present in cultures grown without added extract. $32 \mathrm{~K}$ and $16 \mathrm{~K}$, size markers for $32 \times 10^{3}$ and $16 \times 10^{3} M_{\mathrm{r}}$.

tidergic differentiating activity for cultured sympathetic neurons but also ciliary neurotrophic activity.

To determine whether the factor(s) present in the footpad extracts was antigenically related to CNTF, an antiserum generated against recombinant rat CNTF was used to precipitate the cholinergic activity. Since sciatic nerve contains CNTF (Manthorpe et al., 1986; Lin et al., 1989; Stockli et al., 1989), extracts of sciatic nerve were used to optimize immunoprecipitation conditions. The CNTF antiserum completely precipitated the cholinergic inducing activity from sciatic nerve extracts; the ChAT activity present in cultures treated with sciatic nerve extract from which the CNTF had been precipitated was equivalent to the activity present in control cultures (Fig. 5A). Further, previous studies have shown that the CNTF antiserum completely immunoprecipitates the peptide-inducing activities present in sciatic nerve extract (Rao et al., 1992a). While the CNTF antiserum had no effect on the induction of ChAT by recombinant $\mathrm{CDF} / \mathrm{LIF}$, it precipitated approximately $80 \%$ of the ChAT-inducing activity in the extract from footpads of 21-day-old rats (Fig. 5A). We also tested the ability of the CNTF antiserum to immunoprecipitate the activity in the footpad extract that induces VIP and suppresses NPY levels (Fig. 5B,C). Approximately 50\% of the VIP-inducing activity was precipitated, while only $20 \%$ of the NPY activity was removed. Thus, the CNTF antiserum cross-reacted with a factor(s) present in the footpad extract and immunoprecipitated the majority of the cholinergic inducing activity (see also Rohrer, 1992).

An immunoblot analysis of the extract was performed using the antiserum generated against recombinant rat CNTF to determine if the footpad extract contained authen- 
A.

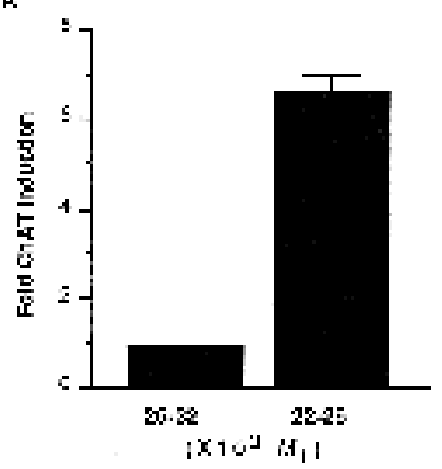

ᄃ

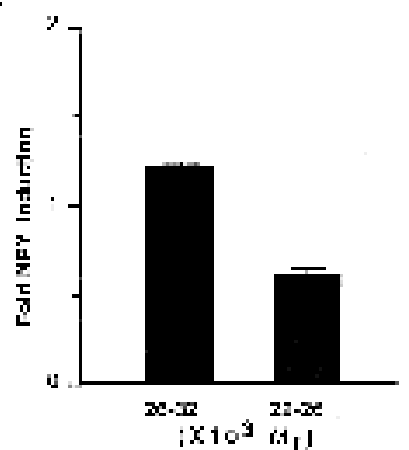

B

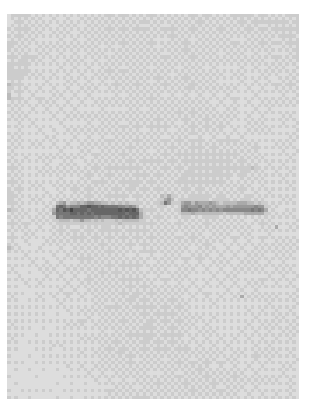

D

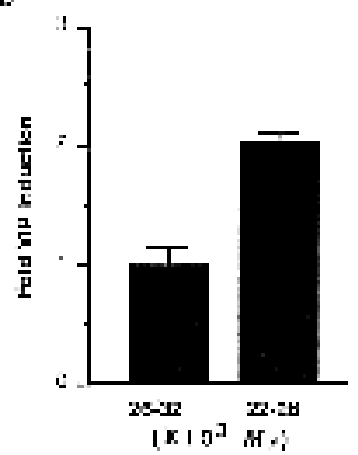

Fig. 3. Regulation of ChAT, TH, NPY, and VIP by SDS-PAGE gel fractions. (A) Purified factor from the Mono P column was subjected to SDS-PAGE, and the fractions corresponding to relative molecular masses of $22-26 \times 10^{3}$ and $26-32 \times 10^{3}$ were eluted and added to cultures of dissociated sympathetic neurons. 7 days after the addition of extracts, neurons were homogenized and aliquots were assayed for ChAT activity. Data are the means of triplicate determinations ( \pm s.e.m.) and are expressed as the fold induction of ChAT activity compared to that present in cultures grown without added extract. (B) Sister cultures treated with SDSPAGE eluates as described above were assayed for TH content by immunoblot. TH content was significantly reduced in cultures treated with the $22-26 \times 10^{3}$ fraction (band on the right) as compared to cultures treated with the $26-32 \times 10^{3}$ fraction (band on the left). (C, D) Sister cultures treated with SDS-PAGE eluates as described above were assayed for NPY (C) and VIP (D) levels by radioimmunoassay. Data are the means of triplicate determinations, \pm s.e.m., and are expressed as the fold induction of neuropeptide immunoreactivity compared to that present in cultures grown without added extract.

tic CNTF. Previous studies had demonstrated that extracts of sciatic nerve and footpads contain similar amounts of cholinergic inducing activity when compared on the basis of extracted protein (Rao and Landis, 1990). For these experiments, three different concentrations of footpad extract and sciatic nerve extract were tested to determine the amounts of each required to yield equivalent induction of ChAT activity: 10, 100 and $250 \mu \mathrm{g}$ of sweat gland extract caused a 2.3-, 8.1- and 15.3-fold induction and 10, 100 and $250 \mu \mathrm{g}$ of sciatic nerve extract caused a 3.5-, 12.6- and 23.5-fold change. Equal amounts of cholinergic inducing activity from extracts of sciatic nerve and footpad were subjected to SDS-PAGE, transferred to nitrocellulose and
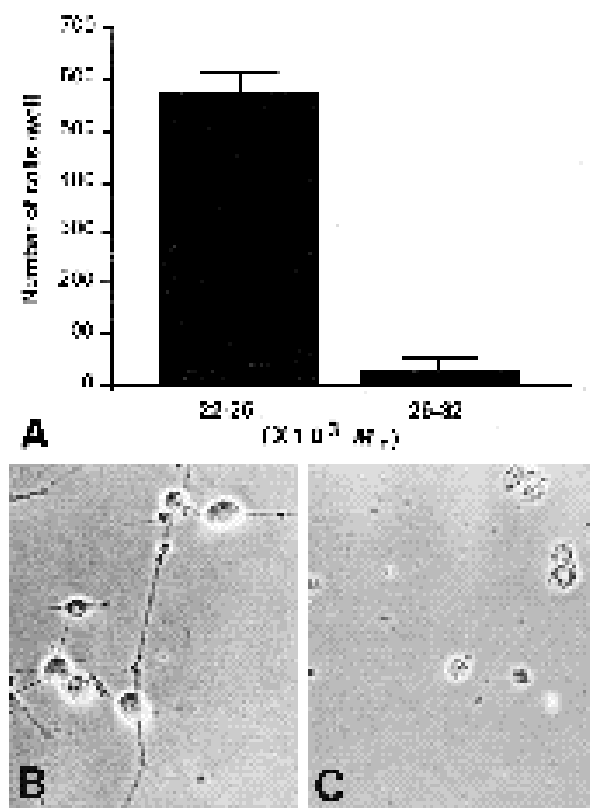

Fig. 4. The purified cholinergic fraction from footpads also has ciliary neurotrophic activity. SDS-PAGE gel eluates corresponding to the $22-26 \times 10^{3} M_{\mathrm{r}}$ and $26-32 \times 10^{3} M_{\mathrm{r}}$ fractions were added to cultures containing freshly dissociated neurons from E8 chick ciliary ganglia. 24 hours later, the cultures were fixed and the number of phase bright cells with neurites was counted. In A, the results are expressed as the number of surviving cells/well \pm s.e.m. and are means of triplicate samples. Phase micrographs of the cultures are shown in B $\left(22-26 \times 10^{3}\right)$ and C $\left(26-32 \times 10^{3}\right)$.

probed with the CNTF antiserum. This procedure yielded a strong signal for recombinant CNTF and a $24 \times 10^{3} \mathrm{Mr}_{\mathrm{r}}$ band in sciatic nerve (Fig. 6A,B). These bands were absent when the antiserum was preincubated with $10 \mu \mathrm{M}$ recombinant CNTF (Fig. 6C). No specific bands were evident in the lanes containing footpad extract which has cholinergic inducing activity (Fig. 6B, lanes 3,4) or hairy skin extract (lane 2) which lacks detectable cholinergic inducing activity (Rao and Landis, 1990). It is important to note that the lanes containing sciatic nerve and footpad extracts, when assayed on cultured sympathetic neurons, contained the same amount of cholinergic inducing activity. Further, we could still detect a band when ten-fold less sciatic nerve extract was loaded. These observations suggest that the major cholinergic activity present in the footpad extracts was not identical to CNTF.

We performed northern blot and in situ hybridization analyses to ascertain whether CNTF mRNA could be detected in footpad. When $30 \mu \mathrm{g}$ of total RNA was probed, a band of $1.3 \mathrm{~kb}$, the expected size of CNTF mRNA (Stockli et al., 1989), was detected in sciatic nerve and a faint band was detected in dorsal root ganglia (Fig. 7). In contrast, no specific binding was evident in lanes containing RNA from footpads or liver. When ten-fold less RNA from sciatic nerve was loaded than from footpad, CNTF message could still be detected in sciatic nerve (Fig. 7B). The same oligonuclotide probe was used for in situ hybridization studies of sciatic nerve and sweat gland (Fig. 8). Many 
Cholinergic neuronal differentiation factors

A
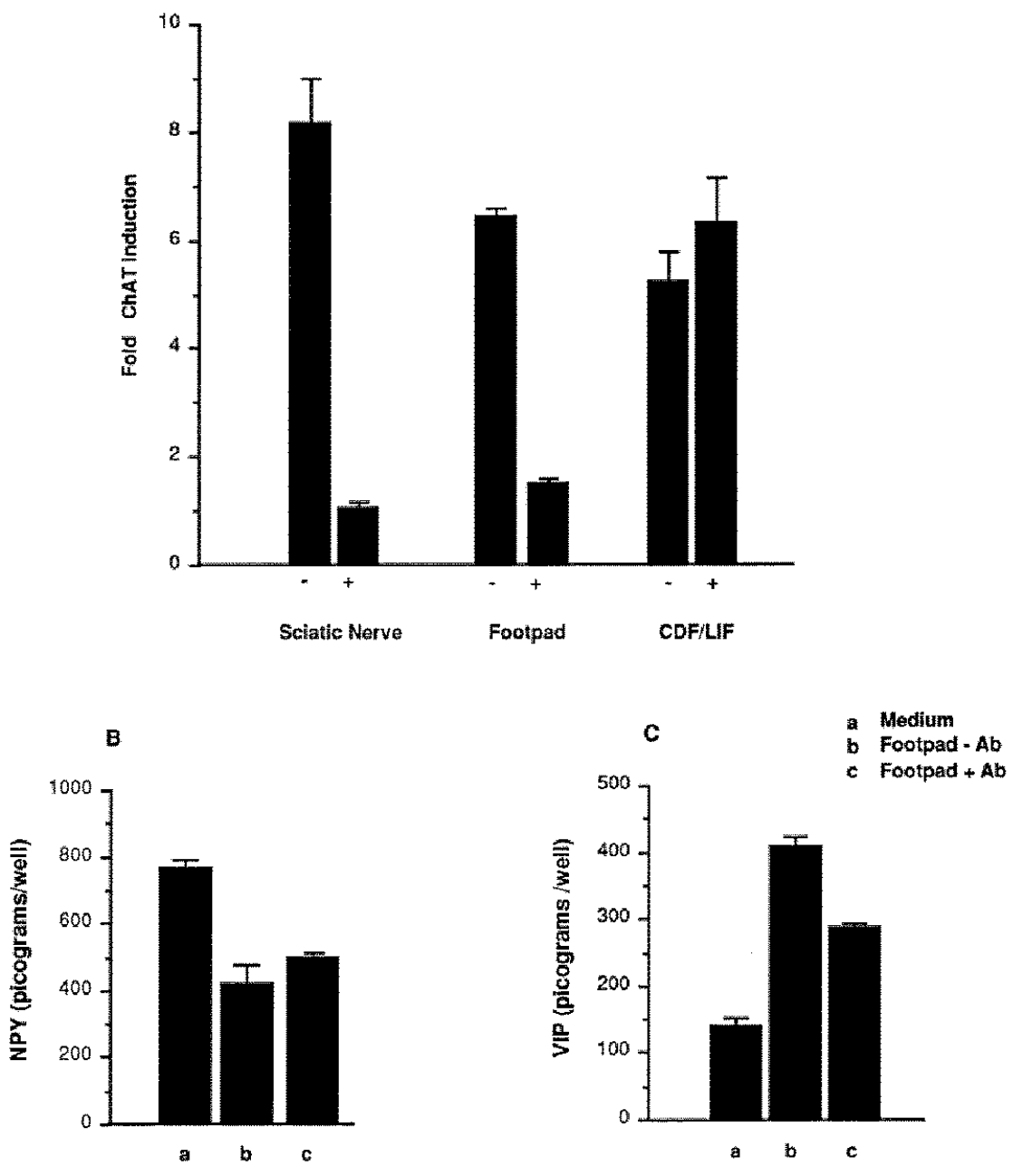

Fig. 5. Antibodies against CNTF partially precipitate cholinergic and neuropeptide differentiation activities from footpad extracts. (A ) Aliquots of sciatic nerve extract from adult rats, footpad extract from 21-day-old rats, and CDF/LIF were incubated with or without polyclonal antiCNTF antibodies. After precipitation of the antibody-antigen complexes with Protein A Sepharose, the supernatants were added to cultured sympathetic neurons. After 8 days, homogenates of the cultured neurons were assayed for ChAT activity. The results are expressed as fold-ChAT induction, mean of triplicate cultures \pm s.e.m., compared to sympathetic neuron cultures grown in medium without extracts. (B,C) Neuron were grown (a) without extracts, or with (50 $\mu \mathrm{g} / \mathrm{ml}$ ) of foot pad extract (crude homogenate) incubated without (b) or with (c) the antibody to CNTF. After 8 days, cultures were assayed for NPY and VIP induction by radioimmunoassay. Results are expressed as picograms of peptide per well, means of triplicate samples \pm s.e.m.

Schwann cells in sciatic nerve exhibited a specific hybridization signal with the antisense probe, while no such signal was apparent with sense control probe (data not shown). No signal was evident in sweat gland tissue with the antisense probe, over either the glands or the Schwann cells investing the sympathetic innervation of the sweat glands.

Since CNTF is known to be present in Schwann cells in sciatic nerve, and footpads from which the extracts were made contain branches of this nerve, we used a polyclonal antiserum generated against a peptide domain of CNTF (Rende et al., 1992) to localize CNTF-like immunoreactivity in footpad (Fig. 9). Sections of sciatic nerve, footpad and hairy skin were incubated with the polyclonal antiserum and specific staining was localized by the peroxidaseantiperoxidase method. As expected, strong staining was observed in a subset of Schwann cells in the sciatic nerve (data not shown; see Rende et al., 1992; Dobrea et al., 1992). In addition, CNTF-immunoreactivity was present in nerve fibers that course through the pad and under the epidermis. This distribution is similar to that of sensory fibers, including those that contain CGRP (Kruger et al., 1989). To determine whether the CNTF-immunoreactivity was, in fact, associated with sensory axons in footpads, double labeling with antisera against CGRP and CNTF was performed. In all cases, the staining patterns were overlapping. CNTF-immunoreactivity was also associated with nerve fibers associated with the epidermis and in the dermis of hairy skin (Fig. 9). No CNTF immunoreactivity was detectable in the sweat gland secretory coil or in the Schwann cells associated with the sympathetic innervation of the sweat glands. Thus, CNTF present in Schwann cells associated with sensory fibers could contribute to the CNTF-like activity present in extracts from footpads. Since cholinergic inducing activity is not present in extracts of hairy skin (Schotzinger and Landis, 1988; Rao and Landis, 1990) which, like footpads, contains nerve bundles with associated CNTF-immunoreactivity, it seems unlikely that the CNTF present in these nerve bundles is the source of the major fraction of the cholinergic inducing activity.

\section{Comparison of the footpad cholinergic factor with $C D F / L I F$}

The size and pI values of the major cholinergic inducing activity in the footpad extract indicated that its biochemical properties were distinct from glycosylated CDF/LIF. It was possible, however, that the footpad factor is a posttranslationally modified form of CDF/LIF that has bio- 


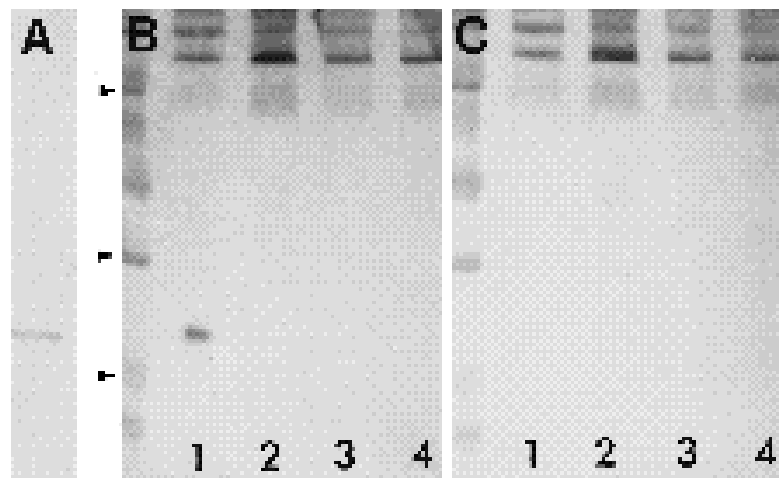

Fig. 6. CNTF is not detectable in immunoblots of extracts of rat footpads. $10 \mathrm{ng}$ of recombinant CNTF (A) and various extracts $(\mathrm{B}, \mathrm{C})$ were subjected to SDS-PAGE, transferred to nitrocellulose, and probed with the anti-CNTF antibodies, with $(\mathrm{C})$ or without $(\mathrm{A}, \mathrm{B})$ preincubation with $10 \mu \mathrm{g}$ recombinant CNTF. Soluble protein (DEAE fractions from adult sciatic nerve extract (lane 1; $60 \mu \mathrm{g}$ ), adult hairy skin extract (lane $2 ; 60 \mu \mathrm{g}$ ), adult footpad extract (lane $3 ; 180 \mu \mathrm{g}$ ) or P21 footpad extract (lane 4; $180 \mu \mathrm{g}$ ) were loaded. The cholinergic inducing activity loaded from sciatic nerve and footpad extracts is equivalent. As expected, the antiserum recognized a $24 \times 10^{3}$ band present in the lane containing authentic CNTF and in the lane containing sciatic nerve extract. The latter band was not detectable if the antibodies were preincubated with CNTF. No bands were detected in lanes containing the other extracts. Arrowheads indicate 92, 30 and $22.5 \times 10^{3} M_{\mathrm{r}}$ size standards.

chemical and immunological properties distinct from those of the protein originally purified from heart-cell-conditioned medium. In addition, the finding that the anti-CNTF antibodies only partially immunoprecipitated the cholinergic inducing activity in footpad extracts suggested that there is more than one cholinergic factor in the footpad extract. To examine whether non-glycosylated CDF/LIF co-chromatographs with the footpad activity, we added recombinant, non-glycosylated CDF/LIF to the footpad homogenate and, after an overnight incubation at $4^{\circ} \mathrm{C}$, determined whether $\mathrm{CDF} / \mathrm{LIF}$ was retained on a DEAE column. As expected (Fukada, 1985), CDF/LIF was not retained on the column, even in the presence of footpad extract (Fig. 10). The footpad factor was, however, retained and no cholinergic inducing activity was detectable in the flow-through fraction (Fig. 10). Thus, the major footpad activity does not behave like either glycosylated or non-glycosylated $\mathrm{CDF} / \mathrm{LIF}$.

To determine if any of the cholinergic inducing activity present in footpad extracts was immunologically related to $\mathrm{CDF} / \mathrm{LIF}$, we employed an antiserum generated against CDF/LIF purified from medium conditioned by rat heart cells (Fukada and Towle, 1992). This antiserum blocks the cholinergic inducing activity of the purified protein on cultured sympathetic neurons (Fig. 11). For these experiments, extracts were made from postnatal day 8 footpads since RTPCR analysis indicated that the highest concentration of CDF/LIF mRNA is present at this age (Yamamori, 1991) and extracts from rat pups 5 days and older contain cholinergic inducing activity (Rao and Landis, 1990). As illustrated in Fig. 11, the antiserum did not block the effects of

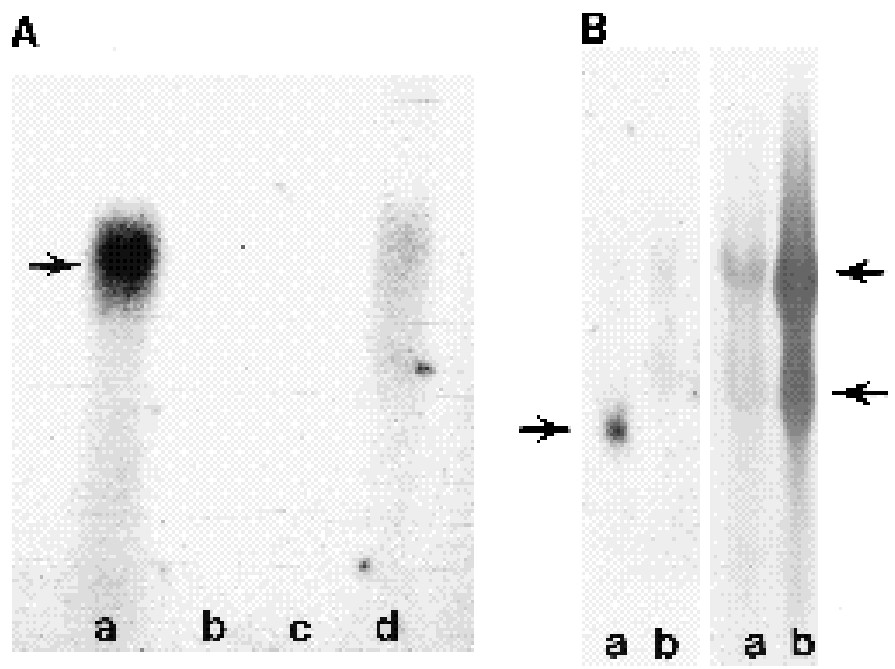

Fig. 7. CNTF mRNA is not detectable in footpad by northern blot analysis. (A) $30 \mu \mathrm{g}$ of total RNA from (a) adult sciatic nerve, (b) footpads, (c) liver and (d) dorsal root ganglia was electrophoresed and transferred onto nylon membrane. The membrane was then probed with a labeled oligonucleotide corresponding to bases 99144 of rat CNTF. The arrow indicates the strong, $1.3 \mathrm{~kb}$ band in sciatic nerve. A fainter band of similar size is present in the dorsal root ganglion lane. No specific signal was detected in the lanes containing either footpad or liver mRNA. Ethidium bromide staining indicated that the mRNA from all four tissues was intact. (B) $2.5 \mu \mathrm{g}$ of total RNA from adult sciatic nerve (lane a) and 25 $\mu \mathrm{g}$ total RNA from footpads (lane b) was probed as described above. The arrow on the left indicates the $1.3 \mathrm{~kb}$ band in sciatic nerve (lane a) but no band is detectable in footpad (lane b). After hybridization, the blot was stained with methylene blue to assess the integrity of the transferred RNA. Arrows on the right indicate the $28 \mathrm{~S}$ and $18 \mathrm{~S}$ bands.

the footpad extract on ChAT activity; it was not significantly different from that of control grown without $\mathrm{CDF} / \mathrm{LIF}$ and the blocking antiserum. In addition, the presence of the antiserum had no effect on the low levels of ChAT activity normally present, or on the induction of ChAT by sciatic nerve extract. These observations are consistent with the failure of antibodies raised against the $\mathrm{N}$ terminal region of CDF/LIF to immunoprecipitate the cholinergic inducing activity in extracts of footpads from 14-day-old and adult rats (Rao and Landis, 1990; our unpublished observations).

\section{Comparison of the footpad and skeletal muscle cholinergic factors}

Skeletal muscle from young rats contains a protein that stimulates the development of ChAT activity in cultures of E14 rat spinal cord (McManaman et al., 1988). This factor has approximately the same relative molecular mass and $\mathrm{pI}$ as the major footpad factor. To assess further the similarity between the factors, we obtained a partially purified (Hap7) fraction of the skeletal muscle factor and tested its ability to alter the neurotransmitter properties of cultured sympathetic neurons. The factor did not alter neuronal survival. No cholinergic induction was observed at any of the concentrations tested and no reduction in $\mathrm{TH}$ was evident at the highest concentration tested (Fig. 12). The same frac- 

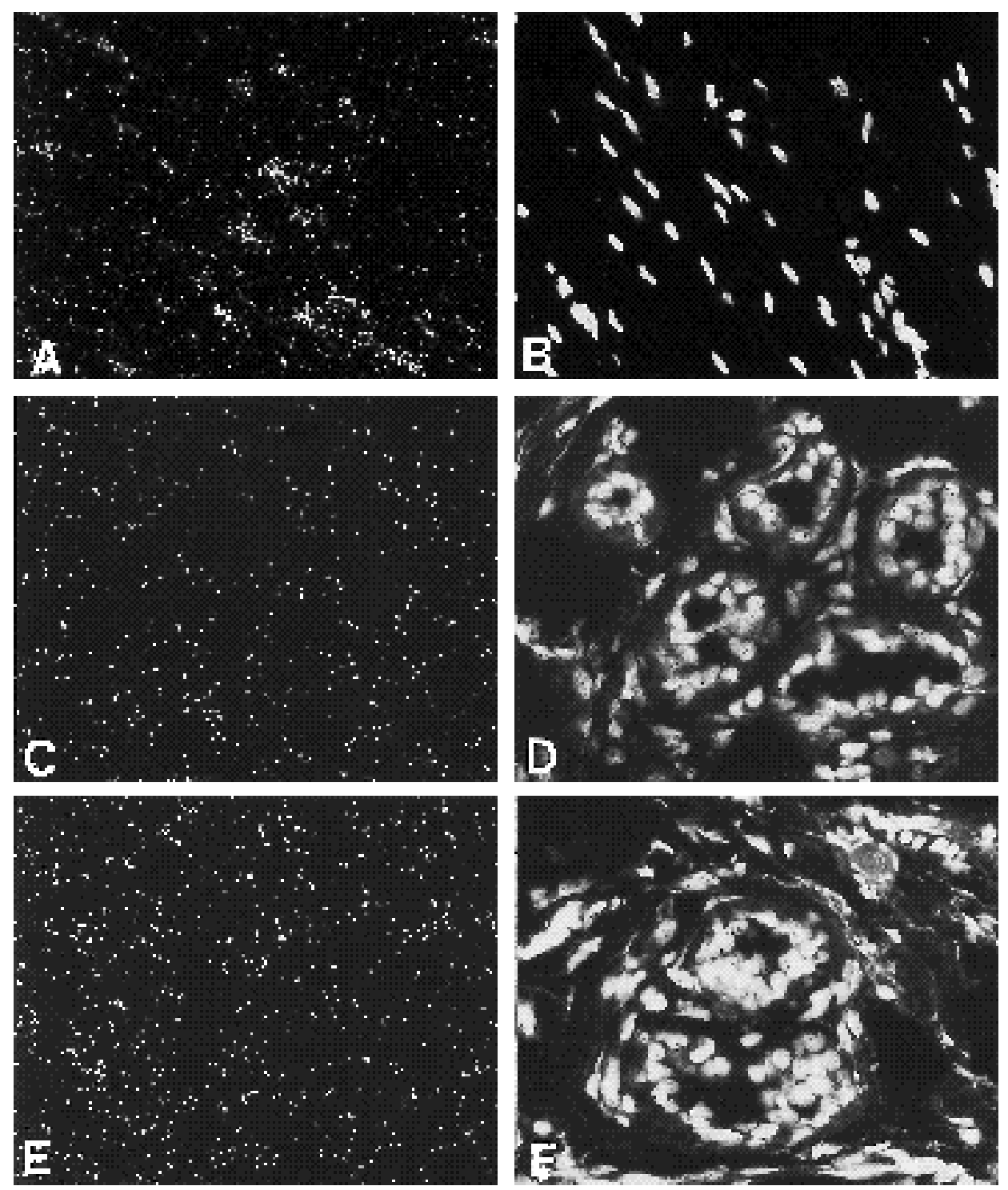

Fig. 8. CNTF mRNA is not detectable in sweat glands by in situ hybridization. Frozen sections of sciatic nerve (A) and sweat gland $(\mathrm{C}, \mathrm{E})$ were probed with either an antisense CNTF oligonucleotide $(\mathrm{A}, \mathrm{C})$ or the corresponding sense oligonucleotide (E). Cell nuclei were visualized with propidium iodide $(\mathrm{B}, \mathrm{D}, \mathrm{F})$. Schwann cells in the sciatic nerve sections specifically bound the sense CNTF probe, while no such binding was detected in sweat glands. $\times 400$. tion elicited a maximal induction of ChAT (150\%) in spinal cord cultures (Dr J. McManaman, personal communication). Therefore, it is unlikely that the footpad and skeletal muscle factor are similar.

\section{Discussion}

We have previously shown that extracts of footpads from both developing and adult rats contain cholinergic differentiation activity when assayed on cultures of neonatal rat sympathetic neurons (Rao and Landis, 1990). Characterization of the extracted factor(s) reveal that the major activity resides in either a single, or several closely related, soluble macromolecules of pI 5.0 and relative molecular masses of $22-24 \times 10^{3}$. We have compared the properties of this major activity to those of three well-characterized cholinergic factors, a cholinergic differentiation factor from skeletal muscle, CDF/LIF and CNTF. A fourth cholinergic factor, MANS, is not a likely candidate for the footpad factor. Unlike MANS (Adler et al., 1989; Wong and
Kessler, 1987), the footpad activity is easily extracted in low salt conditions and no detectable activity is associated with membrane fractions from sweat glands (Rao and Landis, 1990; our unpublished data).

The cholinergic factor from skeletal muscle has a pI of 4.8 and a relative molecular mass of $22 \times 10^{3} M_{\mathrm{r}}$ (McManaman et al., 1988, 1989, 1990). While this factor had not been shown to induce cholinergic function in sympathetic neurons, CDF/LIF enhances survival of cultured motor neurons and increases their levels of ChAT (Martinou et al., 1989, 1992) and CNTF supports motorneuron survival in vitro and in vivo after nerve lesion (Sendtner et al., 1990; Arakawa et al., 1990). Thus, it seemed possible that the muscle-derived cholinergic factor would induce cholinergic function in cultured sympathetic neurons. When we tested a purified fraction of the skeletal muscle factor for ChAT induction under the same conditions in which footpad extract, CDF/LIF and CNTF cause a 10- to 20-fold induction, the skeletal muscle factor did not alter either ChAT activity or TH content. These findings make it unlikely that this factor is responsible for the cholinergic effects of the 

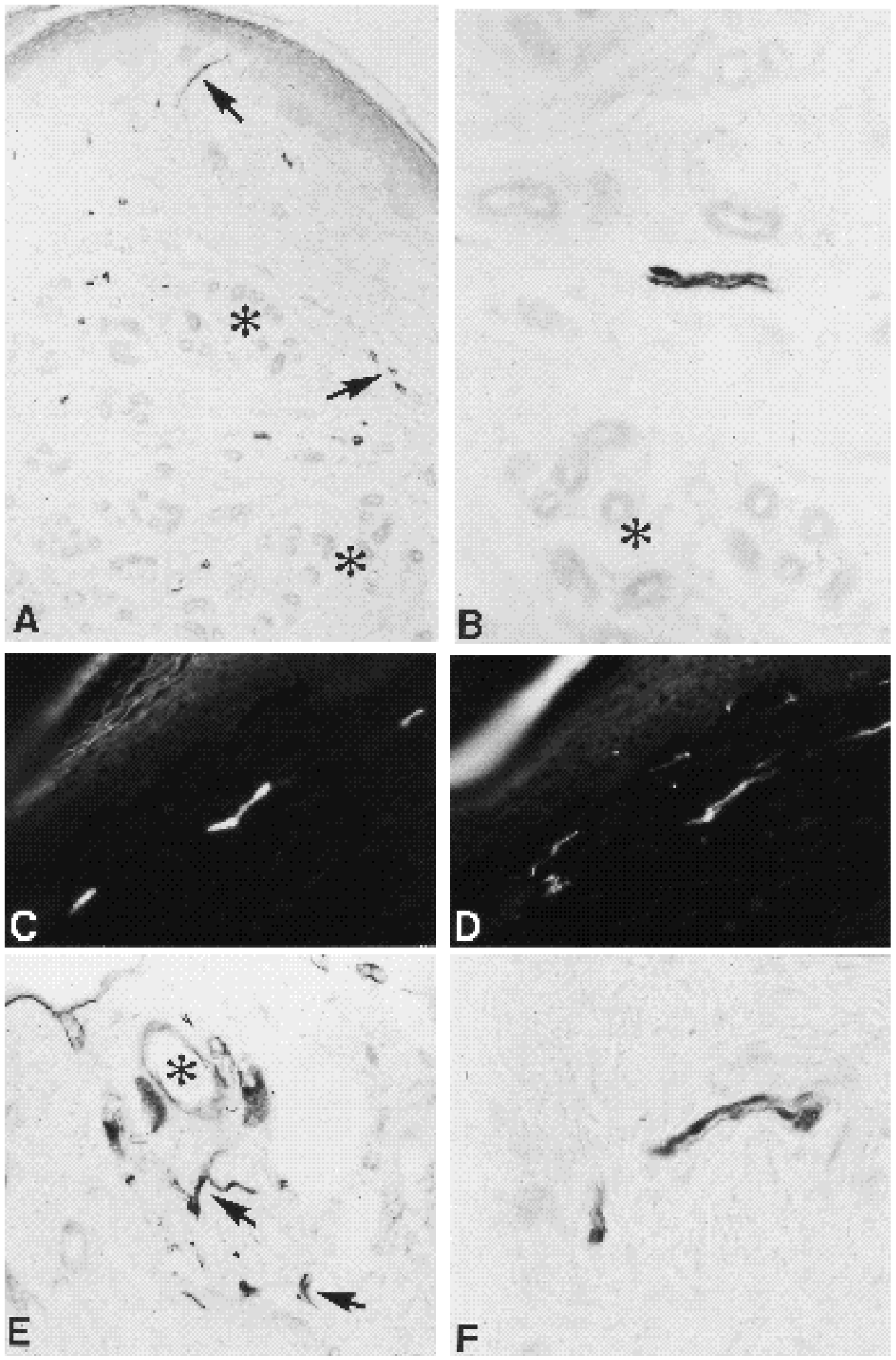

Fig. 9. CNTF

immunoreactivity is absent from sweat glands but present in small nerve fibers in footpad and hairy skin. Frozen sections of sweat glands and hairy skin from adult rats were stained with an antiserum generated against a synthetic peptide sequence from CNTF. In A and $\mathrm{B}$, immunoreactivity was localized with avidinbiotin HRP. The arrows in A indicate several nerve fibers that contain CNTFimmunoreactivity. The middle and lower portions of A contain many sweat gland profiles, two of which are indicated by asterisks. In B, a sweat gland (asterisk) and an adjacent nerve fiber are shown at higher magnification. No specific staining is evident associated with either the sweat glands or their sympathetic innervation. In $\mathrm{C}$ and $\mathrm{D}$, the same section has been doubly labeled for CNTF-immunoreactivity (C) and CGRP (D). The CNTF immunoreactivity present in fibers coursing beneath the epidermis is associated with intensely CGRP-immunoreactive sensory fibers. (E,F) CNTFimmunoreactive nerve fibers, indicated by arrows, are present in hairy skin. The asterisk indicates where a hair has been cut. On either side of the hair shaft are sebaceous glands which possess endogenous peroxidase activity. A $\times 120 ; \mathrm{B} \times 500, \mathrm{C}, \mathrm{D} \times 350, \mathrm{E}$ $\times 150, \mathrm{~F} \times 500$.

footpad extract and, further, they suggest that the cholinergic factor from skeletal muscle is distinct from CNTF.

Two soluble proteins, CDF/LIF and CNTF, exert very similar effects on the expression of neurotransmitter properties by cultured rat sympathetic neurons; they both increase ChAT and VIP expression and reduce $\mathrm{TH}$ and
NPY expression (Ernsberger et al., 1989; Saadat et al., 1989; Yamamori et al., 1989; Nawa and Patterson, 1990; Nawa et al., 1990; Rao et al., 1992a). Each of these effects is also elicited by the purified footpad extract.

$\mathrm{CDF} / \mathrm{LIF}$ is an attractive candidate for the differentiation factor in the footpad extract. Its effects on the neurotrans- 


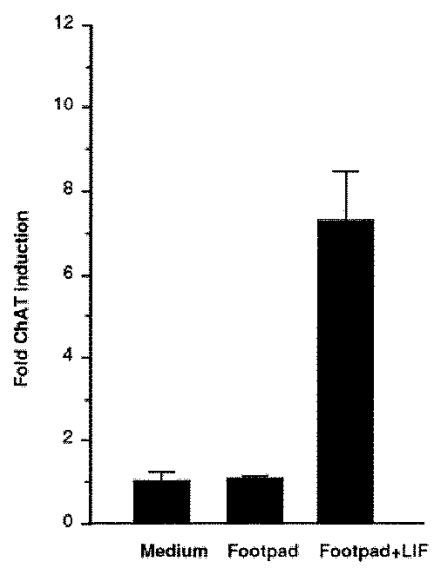

Fig. 10. The major footpad cholinergic activity is biochemically distinct from recombinant CDF/LIF. Extracts from footpads of adult rats $(25 \mu \mathrm{g} / \mathrm{ml})$ were incubated with or without recombinant $\mathrm{CDF} / \mathrm{LIF}(25 \mathrm{ng} / \mathrm{ml})$, and applied to a DEAE column. The flowthrough fraction was collected, concentrated and added to cultured sympathetic neurons. 7 days after the addition of the extracts, samples were assayed for ChAT activity. Results are expressed as fold-ChAT induction ( \pm range) as compared to control cultures grown without extract. The data are means of duplicate cultures; similar results were obtained in two independent experiments.

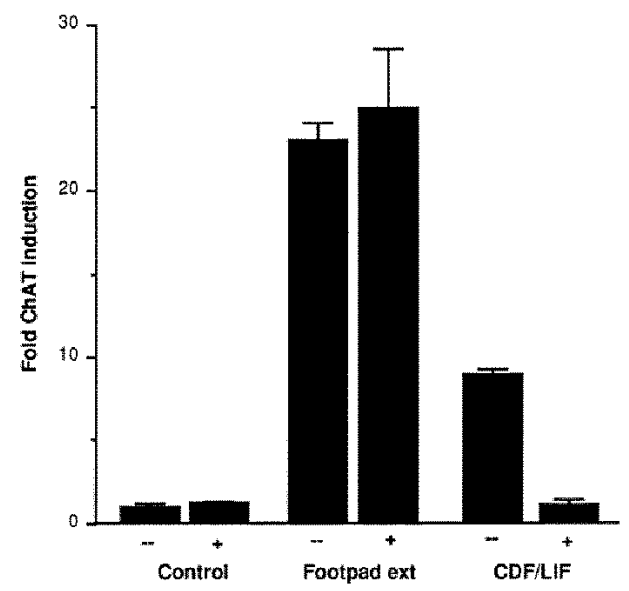

Fig. 11. The cholinergic differentiation activity in sweat gland extracts is not affected by an antiserum that blocks the function of CDF/LIF. Sympathetic neuron cultures were grown either in serum-free medium (control), purified CDF/LIF (20 ng/ml), sciatic nerve extract $(25 \mu \mathrm{g} / \mathrm{ml})$ or extracts from footpads of postnatal day 8 rats $(25 \mu \mathrm{g} / \mathrm{ml})$ with $(+)$ or without $(-) \mathrm{CDF} / \mathrm{LIF}$ antiserum (1:15 dilution). Cultures were assayed for ChAT activity on the ninth day after addition of factors. All samples were run in triplicate and the data are expressed as the mean fold induction \pm s.e.m. Similar results were obtained in three independent experiments.

mitter phenotype of cultured sympathetic neurons are indis tinguishable from those of the purified footpad extract. Moreover, recent experiments using RT-PCR detected a selective expression of CDF/LIF mRNA in P8 footpads; in contrast, CDF/LIF mRNA was not detected in tissues of postnatal rats that are innervated by noradrenergic sympathetic neurons (Yamamori, 1991). Comparison of the bio-

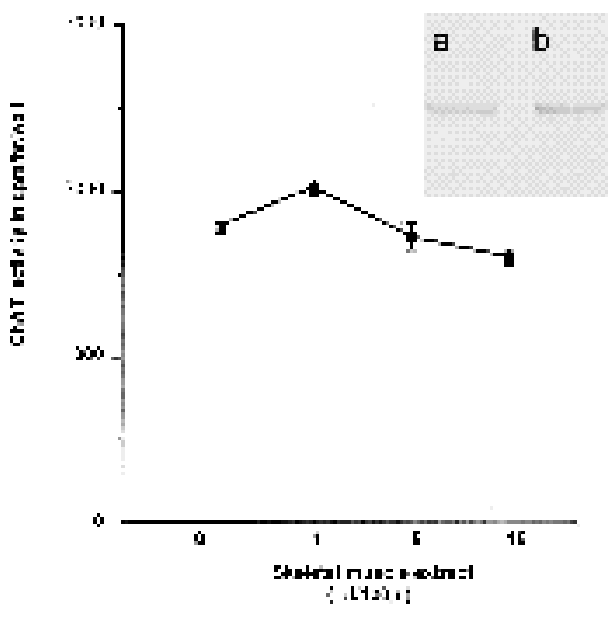

Fig. 12. The cholinergic factor from skeletal muscle does not induce ChAT activity or reduce TH content in cultured sympathetic neurons. Aliquots of the Hap 7 fraction of the skeletal muscle factor were added to sympathetic neuron cultures. 7 days after the addition of the extracts, samples were assayed for ChAT activity. Results are expressed as cts/minute/hour of incubation/well \pm s.e.m. In the inset are shown bands from immunoblots for TH in control (a) and factor-treated (b, $15 \mathrm{ml}$ of extract) cultures. Neuronal numbers for the control cultures and increasing factor doses, prior to extraction, were $1952 \pm 162$; $2077 \pm 15 ; 1993 \pm 28 ; 2033 \pm 26$, respectively. All samples were run in duplicate. Similar results were obtained in three experiments.

chemical properties of CDF/LIF with those of the partially purified footpad factor(s), however, reveals significant differences in size and charge. CDF/LIF purified from heartcell-conditioned medium is a basic $45 \times 10^{3} M_{\mathrm{r}}$ protein while the major cholinergic inducing activity present in the sweat gland extract is an acidic protein with a relative molecular mass in the 22 to $26 \times 10^{3}$ range. In addition, antisera generated against an $\mathrm{N}$-terminal peptide sequence from CDF/LIF failed to immunoprecipitate the cholinergic inducing activity from extracts of footpads from adult rats (Rao and Landis, 1990). The factor in footpad extracts could, however, represent a post-translationally modified form of CDF/LIF. Such modifications could account for the differences in physical properties, as well as the lack of reactivity with antibodies raised against the $\mathrm{N}$-terminal region of $\mathrm{CDF} / \mathrm{LIF}$. A more stringent test for the presence of $\mathrm{CDF} / \mathrm{LIF}$ is the use of an antiserum that directly blocks its activity. The binding of antibodies in such a serum to CDF/LIF would be less likely to be affected by post-translational modifications since the active site of even a modified CDF/LIF should be available for binding to its neuronal receptor. The blocking antiserum did not inhibit the cholinergic activity present in extracts of footpads from postnatal day-8 rat pups, the age at which CDF/LIF mRNA is most abundant (Yamamori, 1991). Thus, while message for CDF/LIF is present, we could not detect any CDF/LIF differentiation activity in the extract. It is possible that $\mathrm{CDF} / \mathrm{LIF}$ is present in the footpad extracts at such a low level that a more stringent assay, for example, immunoprecipitation after separation of the distinct cholinergic inducing activities, is required to detect its presence. 
CDF/LIF present in footpads may also be lost during the extraction.

The biochemical properties of the major footpad cholinergic activity are strikingly similar to those reported for CNTF (Manthorpe et al., 1986; Lin et al., 1989; Stockli et al., 1989). The effects of CNTF and the footpad extract on the neurotransmitter properties of cultured sympathetic neurons are identical (Saadat et al., 1989; Rao and Landis, 1990; Rao et al., 1992a). Moreover, the footpad extract possesses ciliary neurotrophic activity, and this property copurifies with the cholinergic-inducing activity. These findings, coupled with the immunoprecipitation of much of the cholinergic and neuropeptide-regulatory activities by antiCNTF antibodies, provide evidence for the identity of $\mathrm{CNTF}$ as the major cholinergic factor in the footpad extract.

Several lines of evidence, however, are inconsistent with such a conclusion. First, immunoblotting experiments with polyclonal antibodies against rat CNTF failed to reveal CNTF immunoreactivity in footpad extracts. Second, northern blot analysis failed to demonstrate detectable CNTF mRNA in footpads. Third, in immunocytochemical and in situ hybridization studies, neither sweat glands nor the Schwann cells associated with their sympathetic innervation had detectable CNTF immunoreactivity or mRNA. In each case, comparisons were made with sciatic nerve since extracts from sciatic nerve and sweat glands contain equivalent amounts of cholinergic inducing activity (Rao and Landis, 1990) and all the cholinergic inducing activity in sciatic nerve can be immunoprecipitated with CNTF antiserum. Each of the methods yielded positive signals in analyses of sciatic nerve. This is particularly striking in the case of the immunoblot experiments in which equivalent amounts of cholinergic inducing activity from sweat gland were probed and a signal could be detected in tenfold less sciatic nerve extract. It is surprising that the CNTF antiserum that precipitated cholinergic inducing activity from both sciatic nerve and sweat gland extracts recognized a band in the western blot of sciatic nerve extract but not sweat gland. The immunoprecipitation experiments, however, were done with native protein while the immunoblot procedure could have denatured the relevant epitopes. Immunocytochemical studies demonstrate that at least some authentic CNTF should also be present in the extract; a sparse plexus of CNTF-immunoreactive nerve fibers is evident in the footpads, coursing between the glands and beneath the epidermis. The contribution that the CNTF associated with these nerve fibers makes to the cholinergic inducing activity present in the footpad extracts remains to be defined. Since, however, a similar distribution of CNTFimmunoreactive fibers is present in hairy skin, extracts of which lack cholinergic inducing activity, it seems unlikely that the nerve fiber-associated CNTF represents the major source of the differentiation activity present in footpad extracts. Consistent with this interpretation is the observation that extracts of footpads of tabby mutant mice that lack sweat glands (Gruneberg, 1971) but have CNTF-immunoreactive fibers in their footpads contain significantly reduced levels of cholinergic inducing activity (our unpublished observations).

An alternative hypothesis in keeping with our results is that the major footpad factor is a CNTF-like molecule, which is recognized by some, but not all, of the probes used in our studies. Further characterization of the immunoprecipitated material will clarify this issue. Evidence has recently been presented for the existence of such a CNTF homologue in chick (Eckenstein et al., 1990; Leung et al., 1992). This molecule, like mammalian CNTF, lacks a signal peptide but in contrast to mammalian CNTF is secreted into the medium by an unidentified mechanism (Lin et al., 1989; Stockli et al., 1989; Leung et al., 1992). In addition, the presence of an extracellular, soluble CNTF-like molecule has been reported in optic nerve (Hughes et al., 1988; Lillien et al., 1988) but it is not yet clear if this CNTF-like molecule is actually released into the medium (Lillien et al., 1988).

The CNTF antibodies only partially immunoprecipitated the neurotransmitter differentiation activities present in the footpad extract. About $80 \%$ of the cholinergic activity from footpads, and less of the neuropeptide-regulating activities was immunoprecipitated. It is unlikely that this is due to our immunoprecipitation procedures as identical precipitation experiments with sciatic nerve extract precipitated $100 \%$ of the cholinergic inducing and peptide inducing activity (Rao et al., 1992a). Further, independent immunoprecipitation experiments with a distinct antiserum to CNTF yielded a similar result (Rohrer, 1992). These observations suggest that there are two (or more) distinct cholinergic factors present in the footpad extract and that the major component is CNTF-like. The presence of a second cholinergic factor, in principle, complicates the quantitative comparisons between sciatic nerve and footpad which form the basis for excluding authentic CNTF as the major differentiation activity. Even when 10-fold more RNA were probed from sweat glands than from sciatic nerve, however, we did not obtain evidence for the presence of CNTF in footpad.

In summary, we have found that most of the differentiation activity present in footpads of young rats is associated with a molecule(s) that is immunoprecipitated by antibodies that recognize CNTF. While CNTF message and protein were easily detected in northern blot, in situ hybridization and immunoblot analyses of sciatic nerve, we did not detect either in footpads. Some activity remains after immunoprecipitation, suggesting that the extract contains at least two distinct differentiation activities. If two components are indeed present in the extract the second, as yet uncharacterized, component, is unlikely to be CDF/LIF as immunoprecipitation and antibody blocking experiments have failed to detect its presence. Once the differentiation activities in the extract have been identified, their role in the directing the changes that occur in neurotransmitter phenotype in the developing sweat gland innervation in vivo can be defined.

The authors wish to thank Dr Ken Neet for providing NGF, Regeneron Pharmaceuticals for recombinant CNTF and antiCNTF antiserum, Dr Keiko Fukada for purified CDF/LIF and antiserum, Dr B. Samal of Amgen Pharmaceuticals for recombinant human LIF and Dr Chris Beattie for advice. We thank Drs Donna Morissey and Mark Furth of Regeneron Pharmaceuticals for the polyclonal antiserum to CNTF. This work was supported by NINDS Javits Neuroscience Investigator Awards to P.H.P. and S.C.L., a McKnight Foundation Neuroscience Research Award to P.H.P. and an AHA Grant-In-Aid to S.C.L. Dr Rao is the recipi- 
ent of a postdoctoral fellowship from the Northeast Ohio Affiliate of the American Heart Association.

\section{References}

Adler, J., Schleifer, L. and Black, I. B. (1989). Partial purification and characterization of a membrane-derived factor regulating neurotransmitter phenotypic expression. Proc. Natl. Acad. Sci. USA 86, 1080-1083.

Arakawa, Y., Sendtner, M., and Thoenen, H. (1990). Survival effect of ciliary neurotrophic factor (CNTF) on chick embryonic motoneurons in culture: comparison with other neurotrophic factors and cytokines. $J$. Neurosci. 10, 3507-3515.

Barbin, G., Manthorpe, M. and Varon, S. (1984). Purification of chick eye ciliary neurotrophic factor. J. Neurochem. 43, 1468-1478.

Bolton, A. E. and Hunter, W. M. (1973). The labelling of proteins to high specific radioactivities by conjugation to a ${ }^{125} \mathrm{I}$ containing acetylating agent. Biochem. J. 133, 529-539.

Carnow, T. B., Manthorpe, M., Davis, G. E. and Varon, S. (1985). Localized survival of ciliary ganglionic neurons identified neuronotrophic factor gands on nitrocellulose blots. J. Neurosci. 5, 19651971.

Chomczynski, P. and Sacchi, N. (1987). Single-step method of RNA isolation by acid-guanidinium thiocynate-phenol-chloroform extraction. Anal. Biochem. 162, 156-159.

Dobrea, G. M., Unnerstall, J. R. and Rao, M. S. (1992). The expression of CNTF message and immunoreactivity in the central and peripheral nervous system of the rat. Dev. Brain Res. 66, 209-216.

Eckenstein, F. P., Esch, F., Holbert, T., Blacher, R. W. and Nishi, R. (1990). Purification and characterization of a trophic factor for embryonic peripheral neurons: Comparison with fibroblast growth factors. Neuron $\mathbf{4}$, 623-631.

Ernsberger, U., Sendtner, M. and Rohrer, H. (1989). Proliferation and differentiation of embryonic chick sympathetic neurons: Effects of ciliary neurotrophic factor. Neuron 2, 1275-1284.

Fonnum, F. (1969). Radiochemical microassays for the determination of choline acetyltransferase and acetylcholinesterase activities. Biochem. J. 115, 465-472.

Fukada, K. (1985). Purification and partial characterization of a cholinergic differentiation factor. Proc. Natl. Acad. Sci. USA 82, 8795-8799.

Fukada, K. and Towle, M. F. (1992). Function-blocking antibodies generated against cholinergic neuronaldifferentiation factor. Neuroreports, in press.

Fukada, K., Rushbrook, J. I. and Towle, M. F. (1991). Immunoaffinity purification and dose-response of cholinergic neuronal differentiation factor. Dev. Br. Res. 62, 203-214.

Geiss, M.-C. and Weber, M. (1984). Acetylcholine metabolism in rat spinal cord cultures: regulation by a factor involved in the determination of the neurotransmitter phenotype of sympathetic neurons. J. Neurosci. 4, 2269-2278.

Gruneberg, H. (1971). The glandular aspects of the tabby syndrome in the mouse. J. Embryol. Exp. Morph. 25, 1-19.

Hawrot, E. and Patterson, P. H. (1979). Long-term culture of dissociated sympathetic neurons. Methods Enzymol. 58, 574-583.

Hughes, S. M., Lillien, L. E., Raff, M. C. Rohrer, H. and Sendtner, M. (1988). Ciliary neurotrophic factor induces type-2 astrocyte differentiation in culture. Nature 335, 70-73.

Hilton, D. J., Nicola, N. A. and Metcalf, D. (1988). Purification of a murine leukemia inhibitory factor from Krebs ascites cells. Anal. Biochem. 173, 359-367.

Kruger, L., Silverman, J. D., Mantyh, P. W., Sternini, C. and Brecha, N. C. (1989). Peripheral patterns of calcitonin gene-related peptide general somatic sensory innervation: cutaneous and deep terminations. J. Comp. Neur. 280, 291-302.

Landis, S. and Keefe, D. (1983). Evidence for neurotransmitter plasticity in vivo: Developmental changes in properties of cholinergic sympathetic neurons. Dev. Biol. 98, 349-372.

Landis, S. C., Siegel, R. E. and Schwab, M. (1988). Evidence for neurotransmitter plasticity in vivo. II. Immunocytochemical studies of rat sweat gland innervation during development. Dev. Biol. 126, 129140 .

Leblanc, G. and Landis, S. C. (1986). Development of choline acetyltransferase activity in the cholinergic sympathetic innervation of sweat glands. J. Neurosci. 6, 260-265.

Lee, J. M., Adler, J. E. and Black, I. B. (1990). Regulation of neurotransmitter expression by a membrane-derived factor. Exp. Neurol. 108, 109-113.

Leung, D. W., Parent, A. S., Cachianes, G., Esch, F., Coulombe, J. N., Nikolics, K., Eckenstein, F. P. and Nishi, R. (1992). Cloning, expression during development and evidence for release of a trophic factor for ciliary neurons. Neuron 8, 1045-1053.

Lillien, L. E., Sendtner, M., Rohrer, H., and Raff, M. C. (1988). Type-2 astrocyte development in rat brain cultures is initiated by a CNTF-like protein produced by Type-1 astrocytes. Neuron 1, 485-494.

Lin, H. L., Mismer, D., Lile, J. D., Armes, G. L., Butler, E. T., Vannice, J. L. and Collins, F. (1989). Purification, cloning, and expression of ciliary neurotrophic factor (CNTF). Science 246, 1023-1025.

Manthorpe, M., Skaper, S., Williams, L. R. and Varon, S. (1986). Purification of adult rat sciatic nerve ciliary neurotrophic factor. Brain Res. 367, 282-286.

Martinou, J. C., Le Van Thai, A., Cassar, G., Roubinet, F. and Weber, M. J. (1989). Characterization of two factors enhancing choline acetyltransferase activity in cultures of purified motorneurons. $J$. Neurosci. 9, 3645-3656.

Martinou, J. C., Martinou, I. and Kato, A. C. (1992). Cholinergic differentiation factor (CDF/LIF) promotes survival of isolated rat embryonic motoneurons in vitro. Neuron $\mathbf{8 , 7 3 7 - 7 4 4 . ~}$

McManaman, J. L., Crawford, F. G., Stewart, S. S. and Appel, S. H. (1988). Purification of a skeletal muscle polypeptide which stimulates choline acetyltransferase in cultured spinal cord neurons. J. Biol. Chem. 263, 5890-5897.

McManaman, J. L., Crawford, F. G., Clark, S., Richker, J. and Appel, S. H. (1989). Multiple neurotrophic factors from skeletal muscle: demonstration of effects of bFGF and comparisons with the 22-kd CAT development factor. J. Neurochem. 53, 1763-1771.

McManaman, J. L., Oppenheim, R. W., Prevette, D., and Marchetti, D. (1990). Rescue of motoneurons from cell death by a purified skeletal muscle polypeptide: Effects of the ChAT development factor, CDF. Neuron 4, 891-898.

Nawa, H. and Patterson, P. H. (1990). Separation and partial characterization of neuropeptide-inducing factors in heart cell conditioned medium. Neuron 4, 269-277.

Nawa, H., Yamamori, T., Le, T. and Patterson, P. H. (1990). The generation of neuronal diversity: analogies and homologies with hematopoiesis. Cold Spring Harbor Symp. Quant. Biol. 55, 247-253.

Patterson, P. H. and Chun, L. L. Y. (1977). Induction of acetylcholine synthesis in primary cultures of dissociated rat sympathetic neurons. I. Effects of conditioned medium. Dev. Biol. 56, 263-280.

Rao, M. S., Landis, S. C. and Patterson, P. H. (1990). The cholinergic neuronal differentiation factor from heart cell conditioned medium is different from the cholinergic factors present in sciatic nerve and spinal cord. Dev. Biol. 139, 65-74.

Rao, M. S. and Landis, S. C. (1990). Characterization of a target-derived neuronal cholinergic differentiation factor. Neuron $\mathbf{5}, 899-910$.

Rao, M. S., Tyrrell, S., Landis, S. C. and Patterson, P. H. (1992a). Effects of ciliary neurotrophic factor (CNTF) and depolarization on neuropeptide expression in cultured sympathetic neurons. Dev. Bio. 150, 281-293.

Rao, M. S., Patterson, P. H. and Landis, S. C. (1992b). Membraneassociated neurotransmitter stimulating factor (MANS) is very similar to ciliary neurotrophic factor (CNTF). Dev. Biol., in press.

Rende, M., Muir, D., Ruoslahti, E., Hagg, T., Varon, S. and Manthorpe, M. (1992). Immunolocalization of ciliary neurotrophic factor in adult rat sciatic nerve. Glia 5, 25-32.

Rohrer, H. (1992). Cholinergic neuronal differentiation factors: evidence for the presence of both CNTF-like and non-CNTF-like factors in developing rat footpad. Development 114, 689-698.

Rohrer, H., Acheson, A. L., Thibault, J. and Thoenen, H. (1986). Developmental potential of quail dorsal root ganglion cells analyzed in vitro and in vivo. J. Neurosci. 6, 2616-2624.

Saadat, S., Sendtner, M. and Rohrer, H. (1989). Ciliary neurotrophic factor induces cholinergic differentiation of rat sympathetic neurons in culture. J. Cell Biol. 108, 1807-1816.

Schotzinger, R. and Landis, S. C. (1988). Cholinergic phenotype developed by noradrenergic sympathetic neurons after innervation of a novel cholinergic target in vivo. Nature 335, 637-639.

Schotzinger, R. and Landis, S. C. (1990). Acquisition of cholinergic and 
peptidergic properties by the sympathetic innervation of rat sweat glands requires interaction with normal target. Neuron 5, 91-100.

Sendtner, M., Kreutzberg, G. W. and Thoenen, H. (1990). Ciliary neurotrophic factor prevents the degeneration of motor neurons after axotomy. Nature 345, 440-442.

Siegel, R. E. (1989). Localization of neuronal mRNA's by hybridization histochemistry. Meth. in Neurosci. 1, 136-150.

Smith, J. C., Caballito, C. J. and Foldes, F. F. (1967). Choline acetyltransferase inhibitors: a group of styryl-pyridine analogs. Biochem. Pharmacol. 16, 2438-2441.

Stevens, L. M. and Landis, S. C. (1987). Development and properties of the secretory response in rat sweat glands: Relationship to the induction of cholinergic function in sweat gland innervation. Dev. Biol. 123,179-190.

Stevens, L. M. and Landis, S. C. (1990). Target influences on transmitter choice by sympathetic neurons developing in the anterior chamber of the eye. Dev. Biol. 137, 109-124.

Stockli, K. A., Lottspeich, F., Sendtner, M., Masiakowski, P., Carroll, P., Gotz, R., Lindholm, D. and Thoenen, H. (1989). Molecular cloning, expression and regional distribution of rat ciliary neurotrophic factor. Nature 342, 920-923.
Suzuki, H. and Terada, T. (1988). Removal of dodecyl sulfate from protein solution. Anal. Biochem. 172, 259-263.

Varon, S., Manthorpe, M. and Adler, R. (1979). Cholinergic neuronotrophic factors. I. Survival, neurite outgrowth and choline acetyltransferase activity in monolayer cultures from chick embryo ciliary ganglia. Brain Res. 173, 29-45.

White, H. L. and Wu, J. C. (1973). Choline and carnitine acetyltransferases of heart. Biochemistry 12, 841-846.

Wong, V. and Kessler, J. A. (1987). Solubilization of a membrane factor that stimulates levels of Substance P and choline acetyltransferase in sympathetic neurons. Proc. Natl. Acad. Sci. USA 84, 8726-8729.

Yamamori, T. (1991). Localization of CDF/LIF mRNA in the rat brain and peripheral tissues. Proc. Natl. Acad. Sci. USA 88, 7298-7302.

Yamamori, T., Fukada, K., Aebersold, R., Korsching, S., Fann, M-J. and Patterson, P. H. (1989). The cholinergic neuronal differentiation factor from heart cells is identical to leukemia inhibitory factor. Science 246, 1412-1416. 